\title{
That was the year that was
}

The disparate structural themes that have caught the imagination of readers of this, and other journals over the last year provide an apposite illustration of the ever widening impact of structural biology on the efforts of scientists to understand the workings of the cell and the molecular mechanisms that underpin life in general. The uniting principle of the year's research high-lights seems to be just this; diversity. And some of the year's themes are echoed in this year-ending issue of Nature Structural Biology. the structure of the $\mathrm{SH} 3$ domain complexed with peptide ligand in a reversed orientation ${ }^{1,2}$; the tetramerization domain of the antioncogene 553 (ref.3); the ets DNA-binding domain and its interaction with $\mathrm{DNA}^{4}$; and, covered in the News and Views pages, reviews ${ }^{5,6}$ of the recently published hammerhead ribozyme ${ }^{7}$ and GroEL chaperonin structures ${ }^{8}$.

Diversity is, perhaps, what one would expect of a discipline whose practitioners have, through the auspices of recent technological advance, been granted powers beyond the imagination of those who inaugurated the early, pioneering days of the field. Structure determination of non-membrane proteins, while still not a trivial activity, is now often merely a matter of perseverance, although 'merely' may be measured in several years of effort. And there seems little shortage of researchers would who give their eye teeth for a high-resolution three-dimensional structure of their favourite protein or nucleic acid. Furthermore, the unstoppable march of the various genome projects and the seemingly exponential accumulation of previously unknown coding sequences with uncharacterized structure will also fuel the desire for information on structure as part of the quest to detail function. Indeed, the accumulation of gene sequences is outstripping the rate of structure determination at such a rate that there will certainly be a great premium placed on the ability to accurately predict the threedimensional fold of a protein known only by its amino acid sequence. So, in a boom period such as this, it is not surprising that the breadth of the subjects being tackled should be so great.

The award of the 1994 Nobel Prize for Medicine to the discovers of G-proteins, covered in the November editorial, captures one thread of research - signal transduction - that has continued its high profile of recent years. Indeed, G-proteins themselves have featured prominently, with the structure and function of the heterotrimeric G-protein $\alpha$-subunit coming under intense investigation (see, for example, ref. 9 and references therein). The Src homology 3 (SH3) domain has also featured, with the structures of the domain in complex with both synthetic and natural ligands being presented $^{10,11}$. A recent flurry of papers, two of which appear in this issue ${ }^{1,2}$, now shows that the peptide ligand can bind to the same site on different $\mathrm{SH} 3$ domains in opposite orientations. While these results tell us much about the details of the interaction between the $\mathrm{SH} 3$ domain and the poly proline II helical substrate (see the associated News and Views by Saraste and Musacchio $^{12}$ ) the nature of the selectivity of the SH3 domains for different target proteins remains uncertain - indeed, it may be that the $\mathrm{SH} 3$ domain is no more than a passive 'gluing' agent holding components of the signal transduction pathway together and that specificity for a particular signal transduction pathway is conferred by other proteins on the pathway. The first structures of the Pleckstrin Homology domain - associated with proteins involved with signal transduction or the cytoskeletonhave provided intriguing hints about function and the identity of putative ligands, although. what the in vivo ligand is remains to $b \epsilon$ determined ${ }^{13,14}$.

DNA-binding proteins have continued tc make the news (see the March editorial). For example, the structure of the DNA-binding do 
main of p53 - the most commonly mutated gene in human cancers - has added to the cannon of DNA-binding folds and provided a structural rationale for the observed clustering of mutations in the $\mathrm{p} 53$ gene, many of which are implicated in oncogenic progression ${ }^{15}$. p53 functions in vivo as a tetramer and the structure of the tetramerization domain presented in this issue ${ }^{3}$, and an as yet unpublished highresolution crystal structure, differs significantly from that presented earlier in the year ${ }^{16}$. The difference between the structures is in the orientation of the two dimers that interact to form the tetramer; a difference that has important consequences for the spatial disposition of the domains that are attached to either ends of the subunits that comprise the dimers and thence the tetramer. For example, in the light of this new structure it is possible to envision how the p53 may interact with its target binding sites without having to invoke bending of the DNA (which is not seen in vivo). The ets family of proteins have central roles in the development and regulation of tissue systems from Drosophila to humans. The structure of the ets DNAbinding domain from the human Fli-1 protein (chimeras of which are involved in the pathogenesis of Ewing's sarcoma) reveals a helix-turnhelix motif of the catabolite activator protein type $^{4}$. Fli- 1 binds DNA as a monomer and yet does not have the 'winged helix' structure characteristic of other monomeric HTH proteins; HNF-3 for example. It would seem that ets is a new variation of the HTH where the additional contacts with DNA required for monomeric binding are mediated by a 'wing' and a loop rather than two 'wings'.

Knowing the details of the individual components of a system provides the ground work for understanding the whole, nonetheless at some point the components must be brought together and the nature and function of the ensemble of molecules must be grappled with. Signal transduction pathways, for example, involve complex chains of interactions; the fidelity of the transmitted signals must be maintained, signals from different paths may be funnelled into or fan out of molecular junctions', and so on. Similarly, sequence-specific transcription factors must necessarily interact with a whole host of ancillary transcription factors that ultimately either activate RNA polymerase or repress it. One of the problems that will tax structural biologists over the coming year will be the analysis of such multi-component systems. The ideal for some would be to be able to crystallize the various factors caught 'in the act' and in some cases, through hard work and luck, this may be possible. Two large protein complexes - the structures of the F1-ATPase and the chaperonin GroEL (reviewed in refs 17 and 6, respectively) - provide encouragement that such approaches will yield (quite spectacular) results. Not all systems will yield to this type of experimental approach. And these snapshots are, by their very nature, not dynamic. Thus more reductionist approaches will inevitably have to provide many of the details of structure, as well as function (see ref. 7, for example). While perhaps not as immediately'sexy' as the big structure projects, it is certain that structure-driven biochemistry, molecular biology, and the lower resolution structure techniques (cryo-electron microscopy, for example) will have a big part to play in probing the nature of such complex systems.

1. Terasawa et al. Nature struct. Biol. 1, 891-897 (1994).

2. Goudreau, N. et al. Nature struct. Biol.1, 898-907 (1994).

3. Lee, W. et al. Nature struct. Biol. 1, 877-890 (1994).

4. Liang, H. et al. Nature struct. Biol. 1, 871-876 (1994).

5. Pardi, A. Nature struct. Biol. 1, 846-849 (1994).

6. Saibil, H. Nature struct. Biol. 1, 838-842 (1994).

7. Pley, H.W., Flaherty, K.M. \& Mckay, D.B. Nature 372, 68-74 (1994).

8. Braig, K. et al. Nature 371, 578-586 (1994).

9. Spickofsky, N. et al. Nature struct. Biol. 1, 771-781 (1994).

10. Yu et al. Cell 76, 933-945 (1994).

11. Musacchio, A., Saraste, M. \& Wilmanns, M. Nature struct. Biol. 1, 838-842 (1994).

12.Saraste, M. \& Musacchio, A. Nature struct. Biol. 1, 835837 (1994).

13. Wagner, G. Nature struct. Biol. 1, 497-498 (1994).

14. Riddihough, G. Nature struct. Biol. 1, 755-757 (1994).

15. Cho, Y., Gorina, S., Jeffery, P.D. \& Pavletich, N. Science 265, 346-355 (1994).

16. Clore, M. et al. Science 265, 346355 (1994).

17. Capaldi, R. A. Nature struct. Biol. 1, 660-663 (1994). 